Urology

Elsevier Editorial System(tm) for European

Manuscript Draft

Manuscript Number: EURUROL-D-19-00533R1

Title: Updated EAU Guidelines on Renal Cell Carcinoma: immune checkpoint inhibition is the new backbone in first-line treatment of metastatic

clear-cell renal cell carcinoma

Article Type: Brief Correspondence

Section/Category: Kidney Cancer (KID)

Keywords: renal cell carcinoma; metastatic; immune checkpoint inhibitors; ipilimumab; nivolumab; sunitinib; pazopanib; cabozantinib; guidelines; European Association of Urology (EAU)

Corresponding Author: Ms. Karin Plass, MA, Ph.D.

Corresponding Author's Institution: European Association of Urology

First Author: Karin Plass, MA, Ph.D.

Order of Authors: Karin Plass, MA, Ph.D.; Laurence Albiges, M.D., Ph.D.; Tom Powles, M.D., Ph.D.; Michael Staehler, M.D., Ph.D.; Karim Bensalah, M.D., Ph.D.; Rachel H Giles, M.D., Ph.D.; Milan Hora, M.D., Ph.D.; Markus A Kuczyk, M.D., Ph.D.; Thomas B Lam, M.D., Ph.D.; Börje Ljungberg, M.D., Ph.D.; Lorenzo Marconi, M.D., Ph.D.; Axel S Merseburger, M.D., Ph.D.; Alessandro Volpe, M.D., Ph.D.; Yasmin Abu-Ghanem, M.D., Ph.D.; Saeed Dabestani, M.D., Ph.D.; Sergio Fernández-Pello Montes, M.D., Ph.D.; Fabian Hofmann, M.D., Ph.D.; Teele Kuusk, M.D., Ph.D.; Rana Tahbaz, M.D., Ph.D.; Axel Bex, M.D., Ph.D. 


\section{Updated EAU Guidelines on Renal Cell Carcinoma: immune checkpoint inhibition is the new backbone in first-line treatment of metastatic clear-cell renal cell carcinoma}

Laurence Albiges $^{\mathrm{a}}$, Tom Powles ${ }^{\mathrm{b}}$, Michael Staehler ${ }^{\mathrm{c}}$, Karim Bensalah ${ }^{\mathrm{d}}$, Rachel H. Giles ${ }^{\mathrm{e}, \mathrm{f}}$, Milan Hora $^{\mathrm{g}}$, Markus A. Kuczyk ${ }^{\mathrm{h}}$, Thomas B. Lam ${ }^{\mathrm{i}, \mathrm{j}}$, Börje Ljungberg ${ }^{\mathrm{k}}$, Lorenzo Marconi ${ }^{1}$, Axel S. Merseburger ${ }^{\mathrm{m}}$, Alessandro Volpe ${ }^{\mathrm{n}}$, Yasmin Abu-Ghanem ${ }^{\mathrm{o}}$, Saeed Dabestani ${ }^{\mathrm{p}}$, Sergio Fernández-Pello ${ }^{\mathrm{q}}$, Fabian Hofmann ${ }^{\mathrm{r}}$, Teele Kuusk ${ }^{\mathrm{s}}$, Rana Tahbaz ${ }^{\mathrm{t}}$, Axel Bex ${ }^{\mathrm{u}, \mathrm{v}}$

${ }^{a}$ Department of Cancer Medicine, Gustave Roussy, Université Paris-Saclay, Villejuif, France;

${ }^{\mathrm{b}}$ The Royal Free NHS Trust and Barts Cancer Institute, Queen Mary University of London, London, UK.

${ }^{c}$ Department of Urology, Ludwig-Maximilians University, Munich, Germany;

${ }^{\mathrm{d}}$ Department of Urology, University of Rennes, Rennes, France;

${ }^{\text {e}}$ Patient Advocate, International Kidney Cancer Coalition (IKCC), Duivendrecht, the Netherlands;

${ }^{\mathrm{f}}$ Department of Nephrology and Hypertension, Regenerative Medicine Center, University Medical Centre

Utrecht, Utrecht, The Netherlands;

${ }^{g}$ Department of Urology, University Hospital Plzeň and Faculty of Medicine in Plzeň, Charles University, Czech Republic;

${ }^{\mathrm{h}}$ Department of Urology and Urologic Oncology, Hannover Medical School, Hannover, Germany;

${ }^{\mathrm{i}}$ Department of Urology, Aberdeen Royal Infirmary, Aberdeen, UK;

${ }^{\mathrm{j} A c a d e m i c}$ Urology Unit, University of Aberdeen, Aberdeen, UK;

Department of surgical and perioperative sciences, Urology and andrology, Umeå University, Umeå, Sweden

${ }^{\mathrm{l}}$ Department of Urology, Coimbra University Hospital, Coimbra, Portugal;

${ }^{\mathrm{m}}$ Department of Urology, University Hospital Schleswig-Holstein, Lübeck, Germany;

${ }^{\mathrm{n}}$ Division of Urology, Maggiore della Carita 'Hospital, University of Eastern Piedmont, Novara, Italy;

${ }^{\circ}$ Department of Urology, Chaim Sheba Medical Center, Tel-Hashomer, 52621, Ramat-Gan, Israel;

${ }^{\mathrm{p}}$ Department of Clinical Sciences Lund, Lund University, Skåne University Hospital, Malmö, Sweden;

${ }^{\mathrm{q}}$ Department of Urology, Cabueñes Hospital, Gijón, Spain;

${ }^{\mathrm{r}}$ Department of Urology, Sunderby Hospital, Sunderby, Sweden;

${ }^{\mathrm{s}}$ Department of Urology, Royal Free Hospital, Pond Street, London, NW3 2QG, UK;

${ }^{\mathrm{t}}$ Department of Urology, Elbe Kliniken Stade, Stade, Germany;

uepartment of Urology, The Netherlands Cancer Institute, Antoni van Leeuwenhoek Hospital, Amsterdam, The Netherlands;

v Specialist Centre for Kidney Cancer, Royal Free London NHS Foundation Trust and UCL Division of Surgery and Interventional Science, London, UK.

\section{Corresponding author:}

Axel Bex, MD, PhD

Department of Urology, The Netherlands Cancer Institute, Antoni van Leeuwenhoek Hospital

Plesmanlaan 121, 1066 CX, Amsterdam

The Netherlands

Phone: +31 205122553

Fax: +31205122554

E-mail: a.bex@nki.nl

\section{Keywords:}

renal cell carcinoma; metastatic; immune checkpoint inhibitors; ipilimumab; nivolumab; sunitinib; pazopanib; cabozantinib; guidelines

Document: 1394 words 


\begin{abstract}
:
Recent randomized trials have demonstrated a survival benefit for a front-line ipilimumab and nivolumab combination therapy, and pembrolizumab and axitinib combination therapy in metastatic clear-cell renal cell carcinoma. The European Association of Urology (EAU) Guidelines Panel has updated its recommendations based on these studies.

Patient Summary: Pembrolizumab plus axitinib is a new standard of care for patients diagnosed with kidney cancer spread outside the kidney and who did not receive any prior treatment for their cancer (treatment-naïve). This applies to all risk groups as determined by International Metastatic Renal Cell Carcinoma Database Consortium (IMDC) criteria.
\end{abstract}

\title{
Introduction
}

Immune checkpoint inhibitors, including programmed death 1 inhibitors (PD-1), programmed death ligand 1 inhibitors (PD-L1) and cytotoxic T-lymphocyte-associated antigen 4 (CTLA-4) inhibitors have activity in metastatic clear-cell renal cell carcinoma (mRCC). Four studies investigating these combinations in the front-line mRCC setting have recently been published (table 1) [1-4]. These have had a major impact on the EAU guidelines which are updated in this article.

\section{Data influencing new guidelines}

The CheckMate 214 study was the first of these trials to report a superiority of nivolumab and ipilimumab over sunitinib. The primary endpoint focused on the intermediateand poor-risk population according to International Metastatic Renal Cell Carcinoma Database Consortium (IMDC), where the combination demonstrated an overall survival (OS) benefit (HR $0.6395 \%$ CI 0.44-0.89) and led to regulatory approval [1]. This led to a paradigm shift in the treatment of the disease and a change in the EAU guidelines in 20197 [5]. Results 
from CheckMate 214 further established that the combination of ipilimumab and nivolumab was associated with higher response rates (RR) (39\%), complete response (CR) rates (8\%) and duration of response compared to sunitinib. Progression-free survival (PFS) did not achieve the pre-defined endpoint. The exploratory analysis of data in the PD-L1 positive population revealed an OS $\underline{\mathrm{HR}}$ of 0.45 (95\%_CI: 0.29-0.71). Frequency of grade 3-4 adverse events and quality of life (QoL) data favoured the immune combination. The frequency of steroid use has generated controversy, and further analysis as well as real-world data are required. A recent update with 30 months of data showed ongoing benefits for the immune combination with investigator-assessed CR rates of $11 \%$ and an OS HR in the IMDC intermediate- and poor-risk group of 0.66 (95\%CI 0.54-0.80) [6]. The IMDC good-risk group continues to perform better with sunitinib although this appears less pronounced than in earlier analysis (HR for OS 1.22 (95\% CI 0.73-2.04). For these reasons the guideline panel continues to recommend ipilimumab and nivolumab in the intermediate- and poor-risk population.

The Keynote-426 trial (NCT02853331) has recently reported results for the combination of pembrolizumab plus axitinib vs. sunitinib in 861 treatment-naïve cc mRCC patients [3]. OS and PFS (assessed by central independent review) in the intention-to-treat (ITT) population were the co-primary endpoints. RR and sub-group analyses of the PD-L1positive patient population were secondary endpoints. The first interim analysis was triggered after at least 7 months of follow-up and 305 events. The trial was ended at the first interim analysis (median follow-up $=12.8$ months) as both primary endpoints were achieved. The median PFS in the pembrolizumab plus axitinib arm was 15.1 months versus 11.1 in the sunitinib arm (HR 0.69; 95\% CI 0.57-0.84; $\mathrm{p}<0.001)$. Median OS has not been reached in either arm, but the risk of death was $47 \%$ lower in the pembrolizumab plus axitinib arm when 
compared to the sunitinib arm (OS HR 0.53; 95\% CI 0.38-0.74; $<<0.0001)$. Response rates were also higher in the experimental arm (59.3\% vs $35.7 \%)$, with efficacy being demonstrated irrespective of IMDC group and PD-L1 status. Treatment-related adverse events ( $\geq$ grade 3 ) occurred in $63 \%$ of patients receiving pembrolizumab and axitinib vs. $58 \%$ of patients receiving sunitinib. Treatment-related deaths occurred in approximately $1 \%$ in both arms.

The JAVELIN 101 trial, a 886-patient phase 3 RCT of avelumab plus axitinib vs. sunitinib was simultaneously published [2]. It met one of its co-primary endpoints (PFS in the PD-L1 positive population at first interim analysis; median follow-up 11.5 months). PFS and OS in the ITT population wereas HR 0.69 (95\% CI 0.56-0.84) and 0.78 (95\% CI 0.55-1.08), respectively. Treatment-related adverse events ( $\geq$ grade 3 ) occurred in $55 \%$ in both arms. The trial is ongoing and OS results are awaited. It is premature to recommend this combination in the absence of a survival signal. The same applies regarding recommendations for the well tolerated combination of atezolizumab and bevacizumab [4]. This combination has also achieved a PFS advantage over sunitinib in the PD-L1 positive population at interim analysis and ITT (HR 0.74 (95\% CI 0.57-0.96), but does not yet have a significant OS advantage (HR $0.81(95 \%$ CI $0.63-1.03)$ in the ITT population. Since OS results are awaited, even this combination cannot currently be recommended.

Cross-trial comparison is not recommended and should be done with caution (table 1). However, there were some inconsistencies across trials, which should be discussed. The proportion of patients across risk groups in the trials were inconsistent, as the ipilimumab plus nivolumab trial focused on the intermediate and poor-risk populations. The geography of recruitment may have an effect on subsequent therapy and the pembrolizumab plus axitinib trial had a large proportion of patients from outside the USA and Western Europe. Response rates and PFS appeared higher for the avelumab plus axitinib and pembrolizumab plus 
axitinib studies than in the ipilimumab plus nivolumab trial. It is possible that $\mathrm{CR}$ rates for ipilimumab plus nivolumab may be higher than pembrolizumab plus axitinib (8\% vs. $6 \%$ at interim analysis), but follow-up remains short and numbers are too small to draw any conclusions at this stage. More mature data for the two most recent studies will address some but not all of these issues.

This leaves, to date, two immune checkpoint inhibitor-based combinations with proven OS benefit as new standards of care for first-line cc mRCC patients (figure 1; table 2). Pembrolizumab plus axitinib was active irrespective of IMDC risk group and PD-L1 status. The combination achieved all three endpoints of RR, PFS and OS (47\% reduced risk of death). Adverse event profile was in line with the control arm while QoL data are awaited. This combination can therefore be recommended as a new standard of care in first line in all IMDC risk groups.

For treatment-naïve IMDC intermediate and poor risk patients, ipilimumab plus nivolumab remains the other standard, with positive RR and OS endpoints (37\% reduced risk of death). High CR rates, positive QoL data and OS enrichment in the PD-L1 positive population (HR 0.45 (95\% CI 0.29-0.71) are attractive features of this combination [7].

The role of vascular endothelial growth factor receptor-tyrosine kinase inhibitors (VEGFRTKI) alone in front-line metastatic RCC has been superseded. Sunitinib, pazopanib, and cabozantinib (IMDC intermediate- and poor-risk disease) remain alternative treatment options for patients who cannot receive or tolerate immune checkpoint inhibition in this setting (figure $1)$.

\section{Monotherapy immune checkpoint inhibitors in the front line setting}


There are no randomised trials to support the use of single-agent pembrolizumab nor nivolumab in the front-line setting. This is a major shortcoming and delayed the approval of the combination of ipilimumab and nivolumab by the European Medicine Agency. Randomised phase II data for atezolizumab vs. sunitinib showed a HR of 1.19 (95\%CI 0.821.71) which did not justify further development as single agents in first-line [8]. Single-arm phase II data for pembrolizumab showed high response rates of 38\% (up to $50 \%$ in PD-L1 + patients) but PFS of 8.7 (95\% CI 6.7 -12.2) [9]. Based on these results and in the absence of a randomised phase III study, single-agent checkpoint inhibitor is presently not recommended as an alternative in the first-line setting.

CLEAR NCT02811861 (pembrolizumab plus lenvatinib vs. lenvatinib plus everolimus vs. sunitinib) and CHECKMATE 9ER NCT03141177 (nivolumab plus cabozantinib vs. sunitinib) are ongoing randomised phase III trials with sunitinib as comparator and may place new VEGFR TKI immune-oncology (IO) combinations in the discussion [10].

\section{Subsequent therapy after progression on front-line IO therapy.}

The impact of front-line immune-checkpoint inhibition on subsequent therapies is unclear. Randomised data on patients with disease refractory to either nivolumab plus ipilimumab or pembrolizumab plus axitinib in a first-line setting are lacking, and available cohorts are limited [11]. Prospective data on cabozantinib and axitinib are available for patients progressing on immune therapy, but these studies do not focus on the front-line setting and merely involve subset analysis, and are too small for definitive conclusions $[12$, 13]. Retrospective data on VEGFR TKI therapy after progression on front-line immune combinations exist but have significant limitations. When considering this data in totality, it is reasonable to conclude that there is some activity but it remains to be defined. It is therefore 
not possible to recommend one VEGFR TKI above another after IOOO-I-based

eombinationtherapy (figure 2). After pembrolizumab plus axitinib combination, changing the

VEGFR TKI at progression is recommended which may be cabozantinib or any other TKI not

previously used (table 3). Data on sequencing immune checkpoint inhibitors after failure of

immune checkpoint inhibitors is lacking and is presently not recommended.

\section{References}

[1] Motzer RJ, Tannir NM, McDermott DF, et al. Nivolumab plus Ipilimumab versus Sunitinib in Advanced Renal-Cell Carcinoma. N Engl J Med 2018;378:1277-90.

[2] Motzer RJ, Penkov K, Haanen J, et al. Avelumab plus Axitinib versus Sunitinib for Advanced Renal-Cell Carcinoma. N Engl J Med. 2019;380:1103-1115.

[3] Rini BI, Plimack ER, Stus V, et al. Pembrolizumab plus Axitinib versus Sunitinib for Advanced Renal-Cell Carcinoma. N Engl J Med. 2019;380:1116-1127.

[4] Motzer RJ, Powles T, Atkins MB, et al. IMmotion151: A Randomized Phase III Study of Atezolizumab Plus Bevacizumab vs Sunitinib in Untreated Metastatic Renal Cell Carcinoma (mRCC). J Clin Oncol. 2018;36:578.

[5] Ljungberg B, Albiges L, Abu-Ghanem Y, et al. European Association of Urology Guidelines on Renal Cell Carcinoma: The 2019 Update. Eur Urol. 2019;75:799-810.

[6] Tannir NMF, Arén Frontera O, Hammers HJ, et al. Thirty-month follow-up of the phase III CheckMate 214 trial of first-line nivolumab + ipilimumab $(\mathrm{N}+\mathrm{I})$ or sunitinib $(\mathrm{S})$ in patients (pts) with advanced renal cell carcinoma (aRCC). J Clin Oncol. 2019;37; abstr 547.

[7] Motzer RJ, Tannir N, McDermott DF, et al. Nivolumab + Ipilimumab (N+I) vs Sunitinib

(S) for treatment-naïve advanced or metastatic renal cell carcinoma (aRCC): results from CheckMate 214, including overall survival by subgroups. JITC. 2017;5:89.

[8] McDermott DF, Huseni MA, Atkins MB, et al. Clinical activity and molecular correlates of response to atezolizumab alone or in combination with bevacizumab versus sunitinib in renal cell carcinoma. Nat Med. 2018;24:749-757.

[9] McDermott DF, Lee JL, Szylik C, et al. Pembrolizumab monotherapy as first-line therapy in advanced clear cell renal cell carcinoma (accRCC): Results from cohort A of KEYNOTE427. J Clin Oncol. 2018;36.

[10] Gill DM, Agarwal N, Vaishampayan U. Evolving Treatment Paradigm in Metastatic Renal Cell Carcinoma. Am Soc Clin Oncol Educ Book. 2017;37:319-329.

[11] Auvray M, Auclin E, Barthelemy P, et al. Second-line targeted therapies after nivolumab-ipilimumab failure in metastatic renal cell carcinoma. Eur J Cancer. 2019;108:3340 .

[12] Choueiri TK, Escudier B, Powles T, et al. Cabozantinib versus Everolimus in Advanced Renal-Cell Carcinoma. N Engl J Med. 2015;373:1814-1823.

[13] Ornstein MC, Pal SK, Wood LS, et al. Prospective phase II multi-center study of individualized axitinib (Axi) titration for metastatic renal cell carcinoma (mRCC) after treatment with PD-1 / PD-L1 inhibitors. J Clin Oncol. 2018;36.

[14] Phillips B, et al. Oxford Centre for Evidence-based Medicine Levels of Evidence. Updated by Jeremy Howick, March 2009 [access date April 2019]. 
https://www.cebm.net/2009/06/oxford-centre-evidence-based-medicine-levels-evidence$\underline{\text { march-2009/ }}$ 
Response to reviewers

Comments to Author:

Reviewer \#1: To the Authors

Title: as the Authors provided new recommendations even for systemic treatments following the first line, the title should be limited to immune check point inhibition, including "clear cell", i.e. "Updated EAU Guidelines on Renal Cell Carcinoma: immune checkpoint inhibition in metastatic clear-cell renal cell carcinoma".

RESPONSE: We thank the reviewer for the suggestion but the main focus of our manuscript is the change in first-line treatment. In order to address the issues of second-line treatments following the new immune checkpoint inhibitor based first-line treatment we discuss briefly further line treatments but this update is primarily meant to discuss the recent publication of the pembrolizumab and axitinib and avelumab and axitinib trials in view of guideline changes. We want to point out that 'clear-cell' was already added to "metastatic renal cell carcinoma" and we believe that our chosen title captures the update appropriately.

Data influencing new guidelines:

-Page 2, line 6 "paradigm shift in the treatment of the disease and a change in the EAU guidelines in 2017 [5]": it is not 2017, but 2019, referring to reference nr 5

RESPONSE: We thank the reviewer, this is indeed 2019.

-Page 3, line 5 "positive population revealed an OS of 0.45 (95\% Cl: $0.29-0.71)$ ": of HR 0.45 maybe; please check

RESPONSE: Indeed, this should be HR. We added ..." revealed an OS HR of 0.45". Thank you

-Page 4, lines 7-10 "It met one of its co-primary endpoints (PFS in the PD-L1 positive population at first interim analysis; median follow-up 11.5 months. PFS and OS in the ITT population was HR 0.69 ( $95 \% \mathrm{Cl} 0.56-0.84)$ and 0.78 (95\% $\mathrm{Cl} 0.55-1.08)$, respectively": is parenthesis necessary after "11.5 months"? Is "was" correct after "PFS and OS" or is "were" better? = "It met one of its co-primary endpoints (PFS in the PD-L1 positive population at first interim analysis; median follow-up 11.5 months). PFS and OS in the ITT population were HR 0.69 (95\% Cl 0.56-0.84) and 0.78 (95\% Cl 0.55-1.08), respectively". Please check

RESPONSE: Thank you. Indeed a parenthesis is necessary after 11.5 months and it should be 'were' instead of 'was'. We changed accordingly.

-Page 4, lines 13-17 "This combination has also achieved a PFS advantage over sunitinib in the PD-L1 positive population at interim analysis and ITT (HR 0.74 (95\% Cl 0.57-0.96), but does not yet have a significant OS advantage (HR 0.81 (95\% Cl 0.63-1.03). Since OS results are awaited, this combination cannot currently be recommended.": please consider to correct as follows, or as you prefer "This combination has also achieved a PFS advantage over sunitinib in the PD-L1 positive population at interim analysis (HR $0.74(95 \% \mathrm{Cl} 0.57$ 0.96), but does not yet have a significant OS advantage (HR 0.81 ( $95 \% \mathrm{Cl} 0.63-1.03$ ) in ITT 
population. Since OS results are awaited, even this combination cannot currently be recommended."

RESPONSE: We thank the reviewer for this suggestion which we have adapted.

-Page 5, lines 11-12 "This combination can therefore be recommended as a new standard of care in all IMDC risk groups.": please consider to add "in first line" as follows: "This combination can therefore be recommended as a new standard of care in first line in all IMDC risk groups."

RESPONSE: Good suggestion which we adapted. Thank you.

-Page 5, lines 17-21 "The role of vascular endothelial growth factor receptor-tyrosine kinase inhibitors (VEGFR- TKI) alone in front-line metastatic RCC has been superseded. Sunitinib, pazopanib, and cabozantinib (IMDC intermediate- and poor-risk disease) remain alternative treatment options for patients who cannot receive or tolerate immune checkpoint inhibition in this setting (figure 1).": TKI have superseded in every risk group, or just in intermediate/poor risk? Because in Figure 1 Sunitinib and Pazopanib have recommendation $1 \mathrm{~b}$ equal to Pembro/Axitinib in favourable risk. Please specify better Monotherapy immune checkpoint inhibitors in the front line setting: no observation

RESPONSE: To answer your question, TKI have indeed be superseded in every risk group. Sunitinib has been the comparator in Keynote 426 and was inferior. It is correct that sunitinib and pazopanib have level $1 \mathrm{~b}$ evidence in figure 1 because this recommendation is grouped under the box "Alternative in patients who cannot receive or tolerate immune checkpoint inhibitors". In these instances, TKI are still the preferred choice and the level of evidence of the pivotal sunitinib trial and COMPARZ was $1 \mathrm{~b}(1 \mathrm{~b}=$ based on one wellperformed RCT).

We believe that our paragraph on immune checkpoint inhibitor monotherapy is clear and concise and reflects accurately that there is no observation. We do not feel this needs further specification.

Subsequent therapy after progression on front-line IO therapy:

-Page 6, line 1 "not possible to recommend one VEGFR TKI above another after IO-IO combination (figure": IO-IO or IO? Please check

RESPONSE: This is indeed not specifically after IO-IO combination, as this can also be IO-TKI combination or - moving away from first-line - even IO monotherapy (after for example nivolumab in second line). We therefore changed the sentence to "...not possible to recommend one VEGFR TKI above another after IO-based therapy".

References:

-Please check if References have been listed according to Eur Urol Guidelines (i.e. Ref nr $1,9,11)$-Please add vol/pages to Ref $\mathrm{nr} 2,3,4,5,6$-Please add abstract number or pages to Ref $\mathrm{nr}$ 9,13 -Please add journal to Ref $\mathrm{nr} 10$. 
RESPONSE: We checked and adapted accordingly. Thank you

Reviewer \#2: Albiges et al have performed a comprehensive review of existing data from KEYNOTE-426 and JAVELIN-101. The resulting recommendations that they have produced are clear and concise; there is no dispute that I have with the rationale for their recommendations, nor with the ultimate recommendations for the group. The rationale for not including axitinib with avelumab may be viewed as contentious but I feel that this was a rational decision based on the lack of survival benefit seen in the study at most recent update.

RESPONSE: We thank the reviewer for the review. Indeed, we did not include avelumab and axitinib in the recommendations due to a lack of OS data.

Reviewer \#3: This update of the EAU guideline on treatment of metastatic renal cancer contextualizes the multiple recently published randomized phase 3 trials in the systemictreatment naive setting evaluating immune checkpoint-blockade based combination approaches. This synthesis of the data is clear, concise, and offers practical information extremely helpful to the practicing clinician. Given the US FDA approval of pembrolizumab/axitinib just yesterday, it is probably timely to include such information.

RESPONSE: We thank the reviewer for the positive review of our update. 
Take home message

Pembrolizumab plus axitinib are recommended as a new standard of care in all IMDC risk groups. For treatment-naïve IMDC intermediate- and poor-risk patients, ipilimumab plus nivolumab remains standard treatment. Sunitinib, pazopanib, and cabozantinib (in IMDC intermediate- and poor-risk disease) are alternative treatment options in patients who cannot receive or tolerate immune checkpoint inhibition in a first-line setting. 
Figures $1-2$

Figure 1. Guidelines Recommendations for first-line therapy

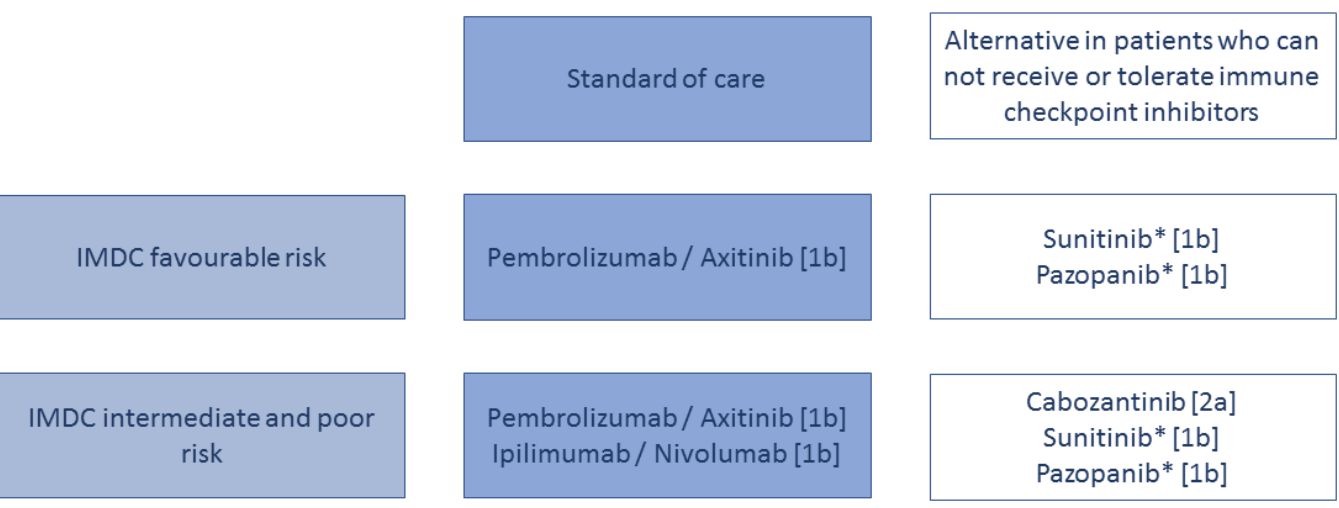

Figure 1: Guidelines Recommendations for first-line therapy

* no OS benefit proven

[ ] = Oxford Level of Evidence [14]

$[1 \mathrm{~b}]=$ based on one randomised controlled phase 3 trial

$[2 \mathrm{a}]=$ based on one randomised controlled phase 2 trial

$[2 \mathrm{~b}]=$ subgroup analysis of a randomised controlled phase 3 trial

$[4]=$ expert opinion

Figure 2. Guidelines Recommendations for later-line therapy

Standard of Care

Any VEGF targeted therapy that

has not been used previously in combination with 10 [4]

Nivolumab [1b]

Cabozantinib [1b]
Alternative

Axitinib* $[2 b]$

Figure 2: Guidelines Recommendations for later-line therapy

* no OS benefit proven

[ ] = Oxford Level of Evidence [14]

$[1 \mathrm{~b}]=$ based on one randomised controlled phase 3 trial

$[2 \mathrm{~b}]=$ subgroup analysis of a randomised controlled phase 3 trial

$[4]=$ expert opinion 



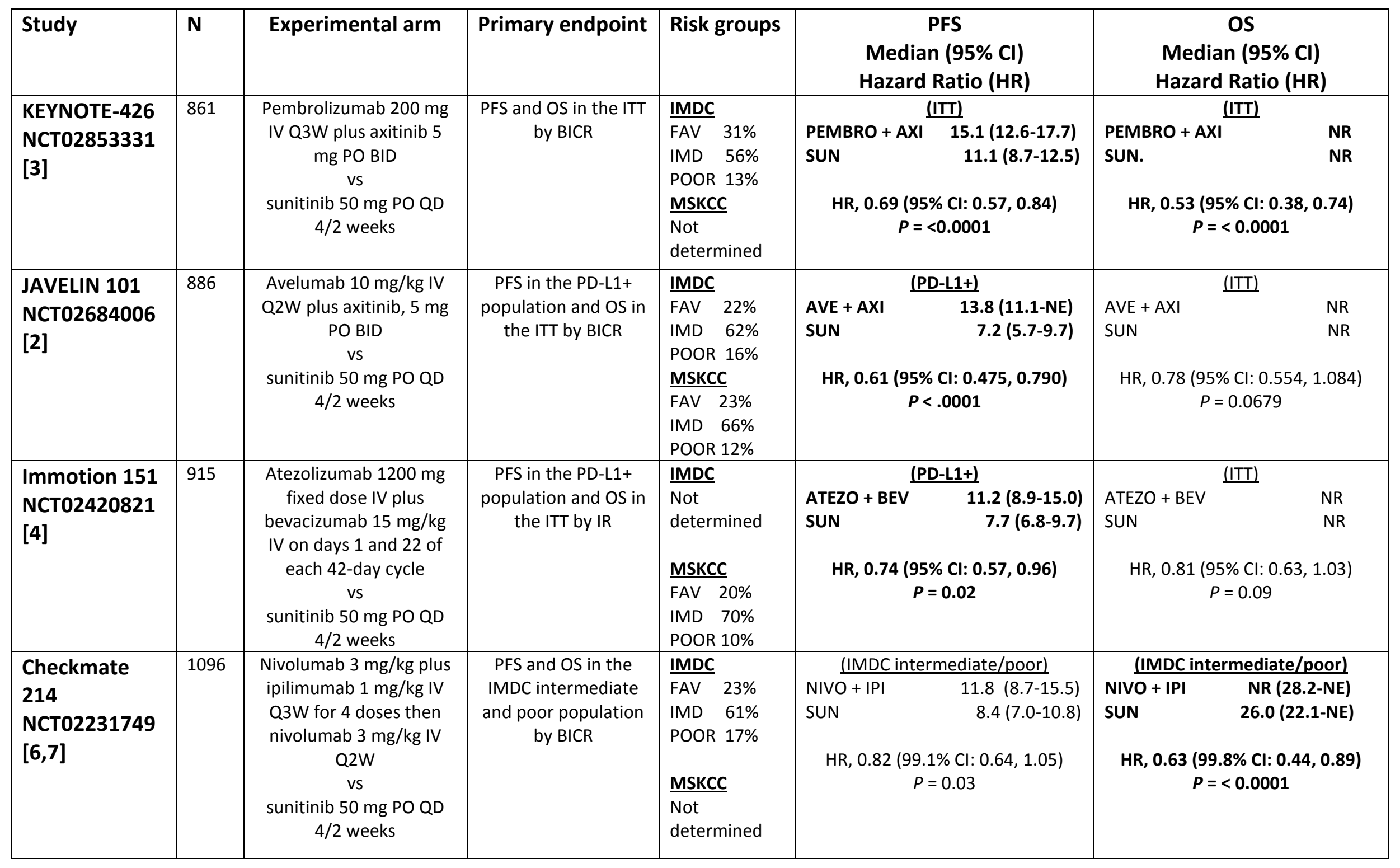


Table 1: Immune checkpoint inhibition combination trials that reported results for the front-line treatment of clear-cell mRCC*

Information under the table:

*PFS and OS results in bold indicate that the primary endpoint has been met.

$B I C R=$ blinded independent central review; $B I D=$ bis in dies (twice daily); $C l=$ confidence interval; $F A V=$ favourable; HR = hazard ratio; $I M D=$ intermediate; IMDC = International Metastatic Renal Cell Carcinoma Database Consortium; IR = investigator review; ITT = intention-to-treat population; $I V=$ intravenous; $O S=$ overall survival; $P D-L 1+=$ programmed death-ligand -1 positive population; $P F S=$ progression free survival; $P O=$ per os (oral); $P O O R=$ poor; $M S K C C=$ Memorial Sloan Kettering Cancer Center; $N=$ number of patients; $N E=$ not estimable; $N R=$ not reached; $Q D=$ daily; $Q 2 W / Q 3 W=$ every second/third week.

\section{Table 2: New recommendations for front-line treatment of metastatic clear-cell RCC}

\begin{tabular}{|l|l|}
\hline Recommendation & Strength rating \\
\hline Offer pembrolizumab plus axitinib to treatment-naive patients with any & Strong \\
\hline $\begin{array}{l}\text { Offer ipilimumab plus nivolumab to treatment-naive patients with IMDC } \\
\text { intermediate - and poor-risk metastatic clear-cell RCC. }\end{array}$ & Strong \\
\hline $\begin{array}{l}\text { Offer sunitinib and pazopanib to treatment-naive patients with IMDC } \\
\text { favourable-, intermediate- and poor-risk metastatic clear-cell RCC }\end{array}$ & Strong \\
\hline
\end{tabular}




\begin{tabular}{|l|l|}
\hline who cannot receive or tolerate immune checkpoint inhibition. & \\
\hline Offer cabozantinib to treatment-naive patients with IMDC intermediate- & Strong* \\
and poor-risk metastatic clear-cell RCC who cannot receive or & \\
tolerate immune checkpoint inhibition. & \\
\hline
\end{tabular}

*While this is based on a randomised phase II trial, cabozantinib (weak) looks at least as good as sunitinib in this population. This justified the same recommendation under exceptional circumstances.

\section{Table 3: New recommendations for treatment after immune checkpoint combination therapy in front-line}

\begin{tabular}{|l|l|}
\hline Recommendation & Strength rating \\
\hline Offer any vascular endothelial growth factor-targeted therapy that has & Weak \\
not been used previously to patients who progress after combination & \\
therapy with immune checkpoint inhibition. & \\
\hline
\end{tabular}


EUROPEAN UROLOGY Authorship Responsibility, Financial Disclosure, and Acknowledgment form.

By completing and signing this form, the corresponding author acknowledges and accepts full responsibility on behalf of all contributing authors, if any, regarding the statements on Authorship Responsibility, Financial Disclosure and Funding Support. Any box or line left empty will result in an incomplete submission and the manuscript will be returned to the author immediately.

Title Prof.Dr.

First Name Axel

Middle Name

Last Name Bex

$\begin{array}{ll}\text { Degree } & \text { M.D., Ph.D. (Ph.D., M.D., Jr., etc.) }\end{array}$

Primary Phone $\quad+3120512$ 2553; (including country code)

Fax Number +31205122554 (including

country code)

E-mail Address a.bex@nki.nl

\section{Authorship Responsibility}

By signing this form and clicking the appropriate boxes, the corresponding author certifies that each author has met all criteria below $(A, B, C$, and $D)$ and hereunder indicates each author's general and specific contributions by listing his or her name next to the relevant section.

$\bigotimes$ A. This corresponding author certifies that:

- the manuscript represents original and valid work and that neither this manuscript nor one with substantially similar content under my authorship has been published or is being considered for publication elsewhere, except as described in an attachment, and copies of closely related manuscripts are provided; and

- if requested, this corresponding author will provide the data or will cooperate fully in obtaining and providing the data on which the manuscript is based for examination by the editors or their assignees;

- every author has agreed to allow the corresponding author to serve as the primary correspondent with the editorial office, to review the edited typescript and proof.

$\bigotimes$ B. Each author has given final approval of the submitted manuscript. 
$\bigotimes$ C. Each author has participated sufficiently in the work to take public responsibility for all of the content.

$\bigotimes$ D. Each author qualifies for authorship by listing his or her name on the appropriate line of the categories of contributions listed below.

The authors listed below have made substantial contributions to the intellectual content of the paper in the various sections described below.

(list appropriate author next to each section - each author must be listed in at least 1 field. More than 1 author can be listed in each field.)

_ conception and design

Prof.Dr. B. Ljungberg

_ acquisition of data
Prof.Dr. A. Bex, Prof.Dr. L. Albiges, Prof.Dr. T. Powles,

Prof.Dr. A. Bex, Prof.Dr. L. Albiges, Prof.Dr. T. Powles,

_ analysis and interpretation of data Prof.Dr. A. Bex, Prof.Dr. T. Powles, Prof.Dr. B.

Ljungberg, Prof.Dr. L. Albiges, Prof.Dr. K. Bensalah, Prof.Dr. R.H. Giles, Prof.Dr. M. Hora, Prof.Dr. M. Kuczyk, Dr. T.B. Lam, Dr. L. Marconi, Prof.Dr. A.S. Merseburger, Prof.Dr. M. Staehler, Prof.Dr.

A. Volpe

_ drafting of the manuscript Prof.Dr. A. Bex

_ critical revision of the manuscript for

important intellectual content Prof.Dr. L. Albiges, Prof.Dr. K. Bensalah, Prof.Dr. R.H. Giles, Prof.Dr. M. Hora, Prof.Dr. M. Kuczyk, Dr. T.B. Lam, Dr. L. Marconi, Prof.Dr. A.S.

Merseburger, Prof.Dr. M. Staehler, Prof.Dr. A. Volpe, Dr. F. Hofmann, Dr. S. Dabestani, Dr. R.

Tahbaz, Dr. S. Fernández-Pello Montes, Dr. Y. Abu-Ghanem, Dr. T. Kuusk

_ statistical analysis

_ obtaining funding

n.r.

_ administrative, technical, or

material support

_ supervision

Ljungberg, Prof.Dr. T. Powles

Prof.Dr. A. Bex, Prof.Dr. L. Albiges, Prof.Dr. B.

_ other (specify) 


\section{Financial Disclosure}

None of the contributing authors have any conflicts of interest, including specific financial interests and relationships and affiliations relevant to the subject matter or materials discussed in the manuscript.

OR

$\bigotimes$ I certify that all conflicts of interest, including specific financial interests and relationships and affiliations relevant to the subject matter or materials discussed in the manuscript (eg, employment/ affiliation, grants or funding, consultancies, honoraria, stock ownership or options, expert testimony, royalties, or patents filed, received, or pending), are the following: (please list all conflict of interest with the relevant author's name):

Prof.Dr. Laurence Albiges has received consulting/advisory fees from BMS, Pfizer, Novartis, Sanofi, Amgen, Bristol-Myers Squibb, Bayer, and Cerulean; and research funding from Pfizer and Novartis.

Prof.Dr. Thomas Powles is a company consultant for Novartis, Pfizer, and GlaxoSmithKline; has received company speaker honoraria from Novartis, Pfizer, GlaxoSmithKline, and Genentech; has participated in trials for GlaxoSmithKline, Pfizer, BMS, Genentech, and Genetech; and has received grants/research support from GlaxoSmithKline, Pfizer, and Novartis.

Prof.Dr. Michael Staehler is a company consultant for Pfizer, Novartis, GlaxoSmithKline, Roche, Astellas, and Bayer; has received company speaker honoraria from Pfizer, Novartis, GlaxoSmithKline, Roche, Astellas, Bayer, and Aveo; has participated in trials for Pfizer, Novartis, GlaxoSmithKline, Roche, Bayer, Aveo, Wilex, and Immatics; has received fellowships and travel grants from Pfizer, Novartis, GlaxoSmithKline, Roche, and Bayer; and has received grants/research support from Pfizer, Novartis, GlaxoSmithKline, Roche, Bayer, and Aveo. In addition, he took part in the S-TRAC trial as an investigator and is an author on the S-TRAC publication.

Prof.Dr. Karim Bensalah has received grants/research support from Pfizer and honoraria or consultation fees from Intuitive Surgical.

Prof.Dr. Milan Hora has received company speaker honoraria from Covidien, Olympus, Janssen, and Astellas; has participated in trials for Janssen; and has received grants/research support from Ipsen.

Prof.Dr. Markus A. Kuczyk is a stock shareholder of Bayer Healthcare, Astellas, Storz, Pfizer, Wyeth, and Novartis; is a company consultant for Karl Storz, Coloplast, AstraZeneca, Astellas, Storz, and Hexal; has received company speaker honoraria from Pfizer, Astellas, Bayer, GlaxoSmithKline, Pierre Fabre, Janssen Cilag, and Hexal; has participated in trials for the ProtecT Study, Millenium Study C21004, Millenium Study C21005, Astellas, Ipsen, and Janssen; and has received grants/research support from Wyeth and Pfizer.

Dr. Thomas B. Lam is a company consultant for and has received company speaker honoraria from Pfizer, GlaxoSmithKline, Astellas, and Ipsen. 
Prof.Dr. Börje Ljungberg has received company speaker honoraria from GlaxoSmithKline, Roche, Pfizer, and Novartis; has participated in trials for GlaxoSmithKline, Medivation, Pfizer, and Janssen R\&D; and has been on advisory boards for Pfizer and GlaxoSmithKline

Prof.Dr. Axel S. Merseburger is a company consultant for Ipsen Pharma, Bayer, Astellas, Janssen Cilag, Novartis, and Pfizer; has received company speaker honoraria from Ipsen Pharma, Wyeth, Astellas, Novartis, Pfizer, and SEP; has participated in trials for AstraZeneca, Bayer, Pfizer, TEVA, Novartis, and Astellas; has received grants/research support from Wyeth; and has participated in a company-sponsored speakers bureau for TEVA, Janssen, Pfizer, Astellas, Ferring, and Novartis.

Prof.Dr. Axel Bex has received company speaker honoraria from Pfizer; has participated in trials for Pfizer Europe; has participated in advisory boards for GlaxoSmithKline and Novartis; is a company consultant for Pfizer and Novartis; and has received grants/research support from Pfizer.

.Prof.Dr. Rachel H. Giles, Prof.Dr. Alessandro Volpe, Dr. Saeed Dabestani, Dr. Fabian Hofmann, Dr. Lorenzo Marconi, Dr. Sergio Fernández-Pello Montes, Dr. Rana Tahbaz and Dr. Yasmin Abu-Ghanem have nothing to disclose.

\title{
Funding Support and Role of the Sponsor
}

I certify that all funding, other financial support, and material support for this research and/or work are clearly identified in the manuscript.

The name of the organization or organizations which had a role in sponsoring the data and material in the study are also listed below:

All funding or other financial support, and material support for this research and/or work, if any, are clearly identified hereunder:

The specific role of the funding organization or sponsor is as follows:

\author{
Design and conduct of the study \\ Collection of the data \\ Management of the data \\ Analysis \\ Interpretation of the data \\ Preparation \\ Review \\ Approval of the manuscript
}

OR 
$\bigotimes$ No funding or other financial support was received.

\section{Acknowledgment Statement}

This corresponding author certifies that:

- all persons who have made substantial contributions to the work reported in this manuscript (eg, data collection, analysis, or writing or editing assistance) but who do not fulfill the authorship criteria are named with their specific contributions in an Acknowledgment in the manuscript.

- all persons named in the Acknowledgment have provided written permission to be named.

- if an Acknowledgment section is not included, no other persons have made substantial contributions to this manuscript.

After completing all the required fields above, this form must be uploaded with the manuscript and other required fields at the time of electronic submission. 
Supplementary file
Click here to download Supplementary file: Supplementary material file.doc

Supplementary file
Click here to download Supplementary file: Supplementary material file.doc

ick here to download Supplementary file: Supplementary material file.doc

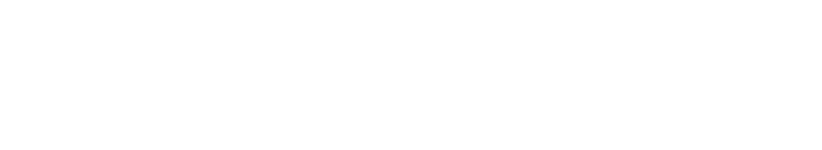

(1)

(1)

(1)

(1)

(1)

(1)

(1)

.

.

.

.

.

.

.

.

.

.

.

.

.

.

.

.

.

.

.

. . 